\title{
Male involvement in family planning use and its determinants in Ethiopia: a systematic review and meta-analysis protocol
}

\author{
Etsay Woldu Anbesu ${ }^{1^{*}}$ (1), Setognal Birara Aychiluhm ${ }^{1}$ and Zinabu Hadush Kahsay ${ }^{2}$
}

\begin{abstract}
Background: The need to include males who require joint spousal decisions is critical in achieving key reproductive health indicators. Low involvement of males in family planning use is one of the contributing factors for low contraceptive use in Ethiopia. Despite this, there are inconsistent findings on the prevalence and determinants of male involvement in family planning use in Ethiopia. Thus, this systematic review and meta-analysis aimed to determine the pooled prevalence of male involvement in family planning use and its determinants in Ethiopia.

Methods: The Preferred Reporting Items for Systematic Reviews and Meta-Analyses guidelines will be used to develop the protocol. The online databases PubMed, CINAHL, Google Scholar, and unpublished gray literature will be searched to retrieve available articles from April 10 to August 11,2021. The two authors will conduct selection of studies, data extraction, and quality assessment. The quality of the studies will be assessed using the Joanna Briggs Institute checklist. The chi-squared test and I-squared statistic will be used to examine heterogeneity among studies. Sources of heterogeneity will be investigated using subgroup analysis and meta-regression based on regions and residence (urban and rural). Publication bias will be examined by observation using funnel plots and statistically by Begg's and Egger's tests. A random-effects model will be used to estimate the pooled prevalence and its determinants of male involvement in family planning use.

Discussion: The role of males in family planning and participation in contraceptive use improves women's uptake and continuity of family planning use. Although there are studies on male involvement in family planning use, there are no synthesis research findings on the pooled prevalence of male involvement in family planning use and its determinants in Ethiopia. Therefore, the findings from this systematic review and meta-analysis will help the national health sector transformational plane emphasize the pooled prevalence and its determinants that drive low male involvement in family planning use in Ethiopia.
\end{abstract}

Keywords: Pooled prevalence, Determinants, Male involvement, Family planning use, Ethiopia

\section{Introduction}

"Male involvement in family planning refers to all organizational activities aimed at men as a discrete group which has the objective of increasing the acceptability

*Correspondence: etsaywold@gmail.com

1 Department of Public Health, College of Medical and Health Sciences, Samara University, Samara, Ethiopia

Full list of author information is available at the end of the article and prevalence of the family-planning (FP) practice of either sex" [1]. Family planning is an effort by a couple to limit or space the number of children they have by using contraceptive methods [2]. Family planning use reduces unwanted pregnancy, induces abortion, and promotes birth spacing. Moreover, it also helps to reduce neonatal, infant, child, and maternal mortality $[2,3]$.

The need to include men who require joint spousal decisions is critical in achieving key reproductive health original author(s) and the source, provide a link to the Creative Commons licence, and indicate if changes were made. The images or other third party material in this article are included in the article's Creative Commons licence, unless indicated otherwise in a credit line to the material. If material is not included in the article's Creative Commons licence and your intended use is not permitted by statutory regulation or exceeds the permitted use, you will need to obtain permission directly from the copyright holder. To view a copy of this licence, visit http://creativecommons.org/licenses/by/4.0/. The Creative Commons Public Domain Dedication waiver (http://creativeco mmons.org/publicdomain/zero/1.0/) applies to the data made available in this article, unless otherwise stated in a credit line to the data. 
indicators [4-10]. However, male roles in couples' fertility decision-making have been given less emphasis. There was a paradigm shift in male involvement and concerns from increasing contraceptive use and attaining demographic goals to gender equality and achieving various reproductive duties since the 1994 International Conference on Population and Development (ICPD) and the 1995 United Nations World Conference on Women $[1,11]$.

Globally, contraceptive method use varies from $69 \%$ in southeast Asia to $11 \%$ in Africa [12]. A qualitative review in sub-Saharan African countries showed low involvement of men in family planning use [13]. To achieve sustainable development goals (SDGs), the participation of men in reproductive health issues is crucial. Moreover, regulating fertility to the level of substitution is essential to increase economic development [14]. Family planning use can avert $32 \%$ and $10 \%$ of maternal and child mortality, respectively $[15,16]$.

Studies have shown that factors that contribute to low family planning coverage include the desire to have more children, lack of knowledge, lack of husband education, and negative perception toward family planning use, sex preference, religious prohibition, and low involvement of men $[9,13,17,18]$.

In Ethiopia, the decision on household-related issues, including fertility, mainly belongs to the husband. The low involvement of men in family planning use is one of the contributing factors for low contraceptive use and high unmet need in Ethiopia. Studies performed in different regions of Ethiopia showed the role of men in family planning use, and male participation in contraceptive use improves women's uptake of family planning services $[2,4,19-21]$. The contraceptive use was low (41.4\%), and there was a high unmet need for family planning (22\%), which contributed to a high total fertility rate (TFR) of 4.6, maternal mortality of 412 per 100,000 live births, neonatal mortality of 30 , infant mortality of 43 , and underfive mortality rate per 1000 live births [2, 22].

Although the Ethiopian government set a target for a contraceptive prevalence rate of $55 \%$ by 2020 to achieve SDGs [23] and develop the National Guideline for Family Planning Services [24], low emphasis has been given to the role of men's involvement. Dissipating this, there is a lack of nationally representative data on male involvement in family planning use in Ethiopia [2, 22]. Several studies have been conducted in different parts of the country on male involvement in reproductive health and utilization of family planning [4, 6, 25-27]. However, there are inconsistent findings on prevalence and its determinants of male involvement in family planning use [16-21, 25]. Therefore, this systematic review and meta-analysis protocol aimed to determine the pooled prevalence of male involvement in family planning use and its determinants in Ethiopia.

\section{Research question}

* What is the pooled prevalence of male involvement in family planning use in Ethiopia?

* What are the determinants of male involvement in family planning use in Ethiopia?

\section{Objectives}

\begin{abstract}
* To determine the pooled prevalence of male involvement in family planning use in Ethiopia

* To identify determinants of male involvement in family planning use in Ethiopia
\end{abstract}

\section{Methods \\ Study protocol and reporting}

A systematic review and meta-analysis protocol will be prepared using the Preferred Reporting Items for Systematic Review and Meta-analyses (PRISMA) guidelines [28]. The PRISMA-P 2015 checklist will be used to report the protocol [29] (Additional file 1).

\section{Eligibility criteria}

All observational studies, including cross-sectional, casecontrol, and cohort studies, will be included. Case reports, case series, conference reports, and expert opinions will be excluded from the review. Studies that reported the prevalence of male involvement in family planning use and its determinants among couples in Ethiopia will be included. Moreover, studies that reported only the prevalence of male involvement in family planning use or at least the measured association between determinant variables on male involvement in family planning use will be included. Studies that only investigate the qualitative approach of male involvement in family planning use will be excluded. If studies address both quantitative and qualitative findings, we will only consider the quantitative findings. Both community- and institution-based studies will be included. Studies published in the English language alone will be included. There will be a restriction on the date of publication since 1994, as this was the period for a paradigm shift in male involvement and concern from increasing contraceptive use and attaining demographic goals to gender equality and achieving various reproductive duties [11]. 


\section{PECO search guide}

\section{Population: Married men}

Exposure: Determinates of male involvement in family planning use. Determinates are exposures that increase or decrease the likelihood of male involvement in family planning use in Ethiopia. The determinates can be marital status, the number of children, discussion with partner, knowledge on contraceptive use, ever used family planning methods, participation in community networks, etc.

Comparison: it is the reported reference group for each determinate in each study: marital status versus single, available children or not, good knowledge versus poor knowledge on contraceptives use, discussion of partners on family planning use or not, etc.

Outcome: The primary outcome of the study will be the pooled prevalence of male involvement in family planning use among married men in Ethiopia. The secondary outcome of the study will be determinates of male involvement in family planning use among married men in Ethiopia. Male involvement in family planning refers to the involvement of males in at least one of the following activities: discussion or spousal communication, support, approval, and contraceptive use of the husband.

\section{Searching strategy and study selection}

Online databases including PubMed, Google Scholar, CINAHL, and unpublished gray literature will be used to search articles from April 10 to August 11, 2021. In addition, cross-reference searching of the included studies will be performed to include related studies. Removal of duplicates and irrelevant studies and inclusion of eligible studies will be performed. The two authors (EW and SB) will independently screen the studies. Studies that mentioned the objective of male involvement in family planning use with full text will be further evaluated for quality. The articles will be retrieved and exported to Endnote version 8 reference manager software to collect and organize search outcomes [30]. The search strategy procedure is shown in the PRISMA diagram (Additional file 2).

The search Medical Subject Heading (Mesh) terms will be developed using the authors' keywords articles and PMID of sample index manuscripts on male involvement in family planning use, titles, and abstracts of studies. Then, search strategies will be developed using different Boolean operators, and modifications will be made based on the types of databases (Additional file 3).

\section{Quality assessments}

Assessment of articles using their title, abstract, and full review of the manuscripts will be performed before the inclusion of articles in the final meta-analysis. The qualities of each article will be assessed by using the Joanna
Briggs Institute Meta-Analysis of Statistics Assessment and Review Instrument (JBI-MAStARI) [31]. Particular attention will be given to a clear statement of the objective, inclusion criteria, study subjects, setting, standard criteria used for measurement of the condition, exposure measurement in validity and reliability strategies to address confounding factors, outcome measurement, and appropriate statistical analysis (Additional file 4). Sensitivity analysis will be conducted to include eligible quality studies in the final systematic review and meta-analysis by investigating the effect size estimates of studies. The quality of the full articles will be assessed by two authors (EW and SB). Any disagreement among reviewers will be resolved by the third author $(\mathrm{ZH})$.

\section{Data extraction and management}

A data extraction template will be constructed in Microsoft Excel (2016) to collect data for the elements of data extraction for full-text eligible manuscripts that will be included in the final systematic review and meta-analysis. The data extraction elements included author name, year of publication, study area, study design, sample size, prevalence or proportion, odds ratio, lower confidence interval, and upper confidence interval. Moreover, new variables will be created to use the elements for analysis using log transformation and standard error on an Excel sheet or STATA 14 after importing the data. Piloting of the data extraction will be carried out before the beginning of the authentic data extraction by all authors. All necessary data will be extracted using the prepiloted Excel data extraction tool. The two authors (EW and SB) will independently extract the data. Any discrepancies will be discussed with a third author $(\mathrm{ZH})$ to reach an agreement. Authors will contact the authors of the studies in case of missing data or incomplete reports.

\section{Data synthesis and analysis}

The extracted data will be imported to STATA version 14 for analysis. The data will be presented using a narrative synthesis of the included studies, and the results will be presented using tables and figures. Square root transformation of data will be performed using the Freeman-Tukey variant of the arcsine to avoid variance variability [32].

The pooled prevalence estimate of male involvement in family planning use in Ethiopia will be performed using a random-effects model [33]. In the random-effects model, we will assume that the true effect size varies from one study to the next and that studies in our analysis will represent a random sample of effect sizes that could have been observed. The summary effect will be our estimate of the mean of these effects. A forest plot will be used to present the pooled prevalence and its determinants of male involvement in family planning use at a statistical significance level of a $p$ value of less than 0.05 
[34]. Heterogeneity across studies will be assessed using Cochran's $Q$ [35] and $I^{2}$ statistics [36]. $I^{2}$ values of $25 \%$, $50 \%$, and $75 \%$ were representative of low, moderate, and considerable heterogeneity, respectively. Subgroup analysis and meta-regression will be performed based on region and residence (urban and rural) to identify the sources of heterogeneity. Moreover, sensitivity analysis will be performed to investigate the effect size estimates of studies. Publication bias will be checked using visual inspection on the funnel plot [34]. An asymmetry of the funnel plot indicates publication bias. Moreover, Egger's and Begg's tests [37] will be conducted to check the potential publication bias, and a $p$ value of $<0.05$ will be used to declare the statistical significance of publication bias.

\section{Discussion}

This systematic review and meta-analysis protocol aims to synthesize the pooled prevalence of male involvement in family planning use and its determinants in Ethiopia. After the 1994 International Conference on Population and Development (ICPD) and the 1995 UN World Conference on Women, attention to male involvement has improved special efforts to emphasize men's joint responsibility and promote their active participation in reproductive health serves $[1,11]$.

Studies have shown that family planning has many benefits, including reducing maternal, child, and infant mortality; protecting unplanned pregnancy; and improving sustainable socioeconomic development. Family planning could avert up to $42 \%$ of maternal mortality [38]. Despite these benefits, contraceptive use is still low, and the unmet need for family planning is high in developing countries, including Ethiopia $[4,7,9,10]$.

Studies in developing countries have examined the role of male involvement in family planning, and male participation improves women's uptake of family planning methods, increases spousal coordination, supports the success of family planning programs, and provides rights to their partners in reproductive health services $[9,13,17,18]$.

Currently, there are no synthesis research findings on the pooled prevalence of male involvement in family planning use and its determinants in Ethiopia. Therefore, this systematic review and meta-analysis protocol will help the development of appropriate strategies that will have an impact on male involvement in family planning use.

This study protocol may have the following limitations. Heterogeneity may exist between studies due to differences in study designs, settings, sample size, and publication biases. Only articles published in the English language will be considered. Moreover, only observational study designs will be included. Studies conducted in hospital or health care settings will not be representative of the general population.

\section{Abbreviations}

FP: Family planning; ICPD: International Conference on Population and Development; JBI-MAStARI: Joanna Briggs Institute Meta-Analysis of Statistics Assessment and Review Instrument; PRISMA: Preferred Reporting Items for Systematic Review and Meta-analyses; SDGs: Sustainable development goals; TFR: Total fertility rate.

\section{Supplementary Information}

The online version contains supplementary material available at https://doi. org/10.1186/s13643-022-01891-x.

Additional file 1. PRISMA-P 2015 checklist.

Additional file 2. Diagrammatic presentation of the studies selection process for systematic review.

Additional file 3. Draft of search strategy to be used using PubMed electronic database.

Additional file 4. JBI critical appraisals for observational studies as shown in the link below https://jbi.global/critical-appraisal-tools.

\section{Acknowledgements}

We would like to thank Samara University for the provision of free Internet and HINARY database website access.

\section{Authors' contributions}

EW conceived, designed, and drafted the systematic review and meta-analysis protocol manuscript. EW, SB, and ZH extensively reviewed and incorporated inputs in the protocol manuscript development. Each author read and approved the final version of the protocol.

\section{Funding}

No founder.

\section{Availability of data and materials}

Additional files for the review protocol were submitted as supplementary materials.

\section{Declarations}

Ethics approval and consent to participate N/A

Consent for publication

Not applicable.

\section{Competing interests}

The authors declare that they have no competing interests.

\section{Author details}

${ }^{1}$ Department of Public Health, College of Medical and Health Sciences, Samara University, Samara, Ethiopia. ${ }^{2}$ School of Public Health, College of Health Science, Mekelle University, Mekelle, Tigray, Ethiopia.

Received: 28 Auqust 2021 Accepted: 21 January 2022

Published online: 01 February 2022

References

1. Federation IPP. Male involvement in family planning utilization; 2011.

2. $\mathrm{FMOH}$, Ethiopia mini demographic and health survey 2019 key indicators

3. Muiga WM. Factors influencing male participation in family planning: a case of Kutus township in Kirinyaga county In Kenya: University of Nairobi; 2014. 
4. Adera A, Belete T, Gebru A, et al. Assessment of the role of men in family planning utilization at Edaga-Hamuse town, Tigray, North Ethiopia. Am J Nurs Sci. 2015:4(4):174-81.

5. Girum T, Shegaze M, Tariku Y. The role of currently married men in family planning and its associated factors in Agaro town, South West Ethiopia. Ann Med Health Sci Res. 2017;7(6).

6. Helamo D, Tessema F, Deribew A. Assessment of husband-wife communication and practice of contraceptives in Angecha woreda, Kembata Tembaro zone, SNNPR, Ethiopia; 2011.

7. Kiogora, C.G., Barriers to male involvement in family planning in Kiambu county, Central Kenya. 2016, University of Nairobi.

8. Mattebo M, Sharma B, Dahlkvist E, et al. Perceptions of the role of the man in family planning, during pregnancy and childbirth: a qualitative study with fifteen Nepali men. J Asian Midwives. 2016;3(1):31-45.

9. Mishra A, Nanda P, Speizer IS, et al. Men's attitudes on gender equality and their contraceptive use in Uttar Pradesh India. Reprod Health. 2014;11(1):1-13.

10. Shisoka J, Litali G. Male involvement in family planning services for population development. J Emerg Trends Econ Manage Sci. 2015;6(7):237-43.

11. Cohen SA, Richards CL. The Cairo consensus: population, development and women. Fam Plan Perspect. 1994;26(6):272-7.

12. Darroch JE, Singh S. Trends in contraceptive need and use in developing countries in 2003, 2008, and 2012: an analysis of national surveys. Lancet. 2013;381(9879):1756-62.

13. Vouking $M Z$, Evina CD, Tadenfok CN. Male involvement in family planning decision making in sub-Saharan Africa-what the evidence suggests. Pan Afr Med J. 2014;19.

14. Doyle MW, Stiglitz JE. Eliminating extreme inequality: a sustainable development goal, 2015-2030. Ethics Int Aff. 2014;28(1):5-13.

15. Mutowo J, Kasu CM, Mufunda E. Women empowerment and practices regarding use of dual protection among family planning clients in urban Zimbabwe. Pan Afr Med J. 2014;17.

16. Tilahun T, Coene G, Temmerman M, et al. Spousal discordance on fertility preference and its effect on contraceptive practice among married couples in Jimma zone, Ethiopia. Reprod Health. 2014;11(1):1-10.

17. Assefa L, Shasho Z, Kasaye HK, et al. Men's involvement in family planning service utilization among married men in Kondala district, western Ethiopia: a community-based comparative cross-sectional study. Contracept Reprod Med. 2021;6(1):1-16.

18. Kassa M, Abajobir AA, Gedefaw M. Level of male involvement and associated factors in family planning services utilization among married men in Debremarkos town, Northwest Ethiopia. BMC Int Health Hum Rights. 2014;14(1):1-8.

19. Bayray A. Assessment of male involvement in family planning use among men in south eastern zone of Tigray, Ethiopia. Sch J Med. 2012;2(2):1-10.

20. Bifato $B$. Assessment of male involvement in family planning use in Loka Abaya district, Southern Ethiopia: cross-sectional study; 2016.

21. Eshete A, Adissu Y. Women's joint decision on contraceptive use in Gedeo zone, Southern Ethiopia: a community based comparative cross-sectional study. Int J Fam Med. 2017;2017.

22. $\mathrm{FMOH}, \mathrm{Central}$ Statistical Agency, Ethiopia demographic and health survey, Addis Ababa. 2016.

23. Nino FS. Sustainable development goals_-United Nations: United Nations Sustainable Development; 2015.

24. $\mathrm{FMOH}$, National guideline final for family planning. 2020.

25. Chekole MK, Kahsay ZH, Medhanyie AA, et al. Husbands' involvement in family planning use and its associated factors in pastoralist communities of Afar, Ethiopia. Reprod Health. 2019;16(1):1-7.

26. Tamiso A, Tassew A, Bekele $H$, et al. Barriers to males involvement in family planning services in Arba Minch Town, Southern Ethiopia: qualitative case study. Int J Public Health Sci. 2016;5(1):46-50.

27. Walle Y, Alamrew Z. The current states of male involvement on family planning and factors correlated with among male factory workers in Bahir Dar City. Am J Public Health Res. 2014;2(5):188-97.

28. Moher D, Liberati A, Tetzlaff J, et al. Preferred reporting items for systematic reviews and meta-analyses: the PRISMA statement. PLoS Med. 2009;6(7):e1000097.

29. Shamseer L, Moher D, Clarke M, et al. Preferred reporting items for systematic review and meta-analysis protocols (PRISMA-P) 2015: elaboration and explanation. Bmj. 2015;349.
30. Bramer W, Bain P. Updating search strategies for systematic reviews using EndNote. J Med Library Assoc. 2017;105(3):285.

31. Munn Z, Tufanaru C, Aromataris E. JBI's systematic reviews: data extraction and synthesis. A J Nurs. 2014;114(7):49-54

32. Lin $L, X u C$. Arcsine-based transformations for meta-analysis of proportions: pros, cons, and alternatives. Health Sci Rep. 2020;3(3):e178.

33. Berkey CS, Hoaglin DC, Mosteller F, et al. A random-effects regression model for meta-analysis. Stat Med. 1995;14(4):395-411.

34. Liu JL. The role of the funnel plot in detecting publication and related biases in meta-analysis. Evid Based Dent. 2011;12(4):121-2.

35. Cooper H, Hedges LV, Valentine JC. The handbook of research synthesis and meta-analysis: Russell Sage Foundation; 2019.

36. Higgins JP, Thompson SG. Quantifying heterogeneity in a meta-analysis. Stat Med. 2002:21(11):1539-58.

37. Egger M, Smith GD, Schneider M, et al. Bias in meta-analysis detected by a simple, graphical test. Bmj. 1997;315(7109):629-34.

38. Khan SA, Amin ZU, Fouzia F, et al. A comparative trial of copper T 380 and Cu 375 IUCD. J Ayub Med Coll Abbottabad. 2010;22(3):185-7.

\section{Publisher's Note}

Springer Nature remains neutral with regard to jurisdictional claims in published maps and institutional affiliations.

Ready to submit your research? Choose BMC and benefit from

- fast, convenient online submission

- thorough peer review by experienced researchers in your field

- rapid publication on acceptance

- support for research data, including large and complex data types

- gold Open Access which fosters wider collaboration and increased citations

- maximum visibility for your research: over 100M website views per year

At BMC, research is always in progress.

Learn more biomedcentral.com/submissions 